\title{
GEOMETRIC REPRESENTATION OF RECURRING SEQUENCES
}

\author{
C. G. LEKKERKERKER \\ Dedicated to Kurt Mahler on his 75th birthday
}

(Received 16 December 1977)

Communicated by J. H. Coates

\begin{abstract}
The asymptotic behaviour of second-order recurring sequences is studied.
\end{abstract}

Subject classification (Amer. Math. Soc. (MOS) 1970): 10 A 35.

The development of the Minkowskian geometry of numbers since the late thirties was vigorously promoted by Professor Mahler. He introduced new fundamental notions, such as that of an automorphic star body (Cassels (1959)), and stressed the geometric aspects of the theory. This was important at a time when the arithmetic nature of the problems treated in this particular branch of number theory was overemphasized. As a matter of fact, geometric intuition often suggests new problems and concepts and even ideas of proofs (Lekkerkerker (1962)).

It is the aim of this note to discuss an elementary problem concerning sequences of real numbers and to show how geometric intuition is helpful in deriving a nontrivial result.

Let $a$ and $b$ be given real numbers. Consider a sequence of real numbers $u_{n}(n=0,1,2, \ldots)$ satisfying the recurrence relation

$$
u_{n}=a u_{n-1}+b u_{n-2} \quad(n \geqslant 2) .
$$

Then $u_{n}$ has the form $\alpha \xi^{n}+\beta \eta^{n}$, where $\xi, \eta$ are the roots of the characteristic equation $x^{2}-2 a x-b=0$ and $\alpha, \beta$ are constants determined by the initial values $u_{0}, u_{1}$. The numbers $\xi, \eta$ are complex conjugate, as well as $\alpha, \beta$. We consider the 
particular case

$$
b=-1, \quad-1<a<1 \text {. }
$$

Then $\xi, \eta$ have absolute value 1. Putting $\xi=e^{i \varphi}, \alpha=\rho e^{i \theta}(\varphi, \theta$ real, $\rho>0)$ we obtain

$$
u_{n}=2 \rho \operatorname{Re} e^{i(\theta+n \varphi)}
$$

Here, $\varphi=\arccos a$. The numbers $\rho$ and $\theta$ can be expressed in terms of $u_{0}, u_{1}, a$. It is easily verified that $\rho$, and therefore also $u_{n}$, is bounded by a fixed constant if $u_{0}, u_{1}$ belong to a fixed bounded interval in $\mathbf{R}$ and $a$ belongs to a fixed subsegment of $(-1,1)$. We remark that formula (1) may be interpreted by saying that $u_{n}$ is obtained from $u_{n-1}, u_{n-2}$ by the following procedure. Take a circle about the origin in the complex plane such that this circle contains two points $w_{n-1}, w_{n-2}$ with real parts $u_{n-1}, u_{n-2}$ and argument difference $\arg w_{n-1}-\arg w_{n-2}=\varphi$, and rotate about an angle $\varphi$. Then the new point $w_{n}$, say, has real part $u_{n}$. If, for given $u_{n-1}, u_{n-2}$, we vary $a$, and therefore the angle $\varphi$, then also the radius of the circle varies.

Let us now modify the problem by admitting $a$ to vary with the index $n$. Then the asymptotic behaviour of the sequence $\left(u_{n}\right)$ may be entirely different. In fact, we have the following:

THEOREM. There exist initial values $u_{0}, u_{1}$ and a sequence of real numbers $a_{n}$ converging to zero such that $\left|u_{n}\right| \rightarrow \infty$ as $n \rightarrow \infty$. Here $u_{n}$ is determined by $u_{0}, u_{1}$ and the recurrence relation $u_{n}=a_{n-1} u_{n-1}-u_{n-2}$.

Proof. We try to obtain the numbers $u_{n}$ as real parts of complex numbers $w_{n}$. Instead of $w_{n}$ we work with triples $w_{n}^{(n-2)}, w_{n}^{(n-1)}, w_{n}^{(n)}$ (for $n=0,1$ we only consider $w_{0}^{(0)}$, and $w_{1}^{(0)}, w_{1}^{(1)}$ respectively) such that the following properties are fulfilled:

(i) $\operatorname{Re} w_{n}^{(n-2)}=\operatorname{Re} w_{n}^{(n-1)}=\operatorname{Re} w_{n}^{(n)}$;

(ii) the numbers $w_{n}^{(n)}, w_{n+1}^{(n)}, w_{n+2}^{(n)}$ lie on a circle about the origin, with angular distance $\varphi_{n+1}=\arccos a_{n+1}$.

We start with $w_{0}^{(0)}=e^{-\pi i / 4}, w_{1}^{(0)}=e^{\pi i / 4}, a_{1}=0$ and continue as indicated in the figure.

In this picture, for simplicity, we have denoted $w_{1}^{(0)}, w_{1}^{(1)}, w_{2}^{(0)}$ by $1,1^{\prime}, 2$ and so on. The points on the four arcs drawn in the figure consecutively have arguments

$$
\left\{\begin{array}{lllll}
-\frac{1}{4} \pi, & \frac{1}{4} \pi, & \frac{3}{4} \pi & & \\
& \frac{1}{4} \pi+\varepsilon, & \frac{3}{4} \pi-\varepsilon, & \frac{5}{4} \pi-3 \varepsilon & \\
& \frac{3}{4} \pi-3 \varepsilon, & \frac{5}{4} \pi-\varepsilon, & \frac{7}{4} \pi+\varepsilon & \\
& & \frac{5}{4} \pi, & \frac{7}{4} \pi, & \frac{9}{4} \pi
\end{array}\right.
$$


apart from second-order effects. This means that $a_{2}, a_{3}, a_{4}$ are so chosen that

$$
\varphi_{2}=\frac{1}{2} \pi-2 \varepsilon, \quad \varphi_{3}=\frac{1}{2} \pi+2 \varepsilon, \quad \varphi_{4}=\frac{1}{2} \pi .
$$

Here, $\varepsilon$ is a small positive quantity. The mentioned second-order effects are of the order $O\left(\varepsilon^{2}\right)$; the values in the first and last line of (2) and the value of $\varphi_{4}$ are exact, while $\varphi_{3}$ is such that in the figure the points $3^{\prime \prime}, 4^{\prime \prime}$ have equal height. At the end of the procedure the radius of the circle has increased by a factor of order $1+c \varepsilon$, $c$ a positive constant.

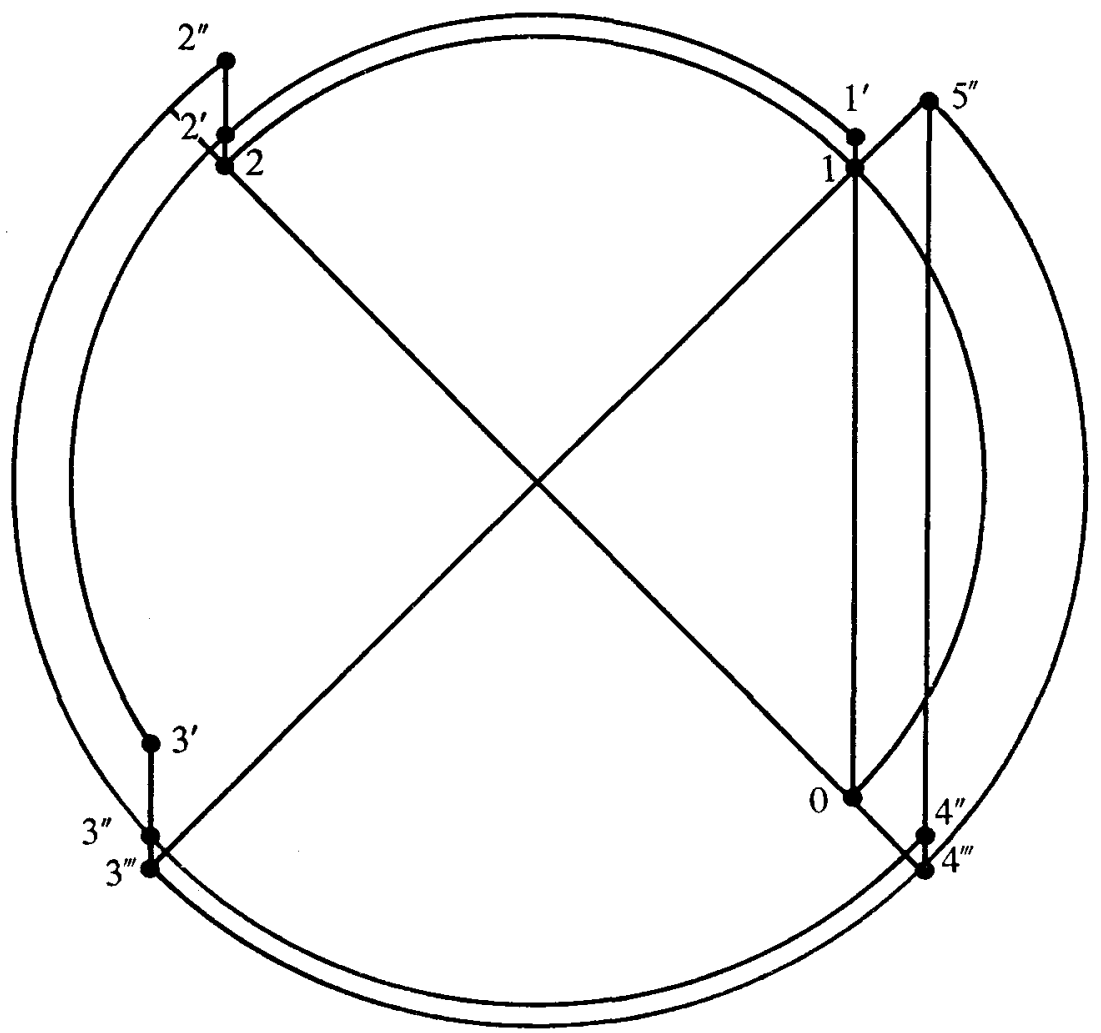

The process described can be repeated. If, in the $n$th revolution, we take $\varepsilon=1 /(n c)$, then the theorem follows because $\Pi(1+1 / n)$ diverges.

From the construction it is clear that we can arrange that $\left|u_{n}\right| \gg e^{n^{\theta}}$, where $\theta$ is any constant with $0<\theta<1$. It is not easy to give conditions in order that the sequence $\left(u_{n}\right)$ be bounded. Roughly speaking, the asymptotic behaviour of this sequence depends on the differences $a_{n}-a_{n-1}$, as well as on the distribution of the partial sums $\sum_{i=0}^{n} a_{i}$. This is clear from the picture. But we shall not elaborate on this point. 


\section{References}

J. W. S. Cassels (1959), Introduction to the Geometry of Numbers (Grundl. Math. Wiss. 99, Springer, Berlin).

C. G. Lekkerkerker (1962), Meetkundige Voorstelling (Assen).

Mathematics Institute

Roetersstraat 15

Amsterdam

Holland 